\title{
Wärmeanwendungen unter Berücksichtigung der Wärmeregulation - Teil 1
}

\author{
Otto Pecher ${ }^{\mathrm{a}, \mathrm{b}}$ Thomas Zeiger Wolfgang Schobersberger $^{\mathrm{d}, \mathrm{e}}$ \\ ${ }^{a}$ Gemeinschaftspraxis für Allgemeinmedizin und Naturheilverfahren, \\ ${ }^{b}$ Dropcon CRO, Aying, Deutschland \\ 'Abteilung für Forschung und Entwicklung, Physiotherm, Vomp, \\ dInstitut für Sport-, Alpinmedizin und Gesundheitstourismus (ISAG), Tiroler Landeskrankenanstalten GmbH (TILAK), Innsbruck, \\ eInstitut für Sport-, Alpinmedizin und Gesundheitstourismus (ISAG), UMIT - Private Universität für Gesundheitswissenschaften, \\ Medizinische Informatik und Technik GmbH, Hall in Tirol, Österreich
}

\section{Schlüsselwörter}

Thermoregulation · Thermoneutralzone · Sauna · Infrarotkabine . Infrarotstrahlung · Hauttemperatur · Hauttemperaturmessung · Erythema ab igne $\cdot$ Verbrennung

\section{Zusammenfassung}

Wärmeanwendungen sind in der Krankheitsprävention und zur Linderung vielfältiger Beschwerden ein fester Bestandteil unserer Kultur. Durch die Fortschritte der Medizin wurden Wärmeanwendungen als therapeutisches Verfahren in Randgebiete verdrängt. Mit dem demographischen Wandel, der Zunahme chronischer und vor allem stoffwechselbedingter Erkrankungen sowie durch den Kostendruck im Gesundheitswesen wächst jedoch das Interesse an diesen Verfahren bei Betroffenen sowie auch in der Medizin. Neben der Konduktion und Konvektion werden im privaten und medizinischen Umfeld radiative Verfahren genutzt. Die Wirkung der Wärmeapplikation ist unabhängig vom Medium mit reflektorischen Reaktionen (Muskelentspannung und Schmerzlinderung) sowie Effekten auf Basis der Durchblutungserhöhung und der Temperaturerhöhung im Gewebe erklärbar. Da Wärme im Körper praktisch ausschliesslich mittels Konvektion über das Blut verteilt werden kann, erklärt sich der Einfluss auf das Herzkreislaufsystem. Unter Berücksichtigung der Wärmeregulationsmechanismen lassen sich drei Prinzipien unterscheiden. Die Wärmeapplikation oberhalb, innerhalb und unterhalb der Thermoneutralzone differieren jeweils hinsichtlich der Herzkreislaufbelastung und der Wärmeverteilung. Mit Wärmeanwendungen sind allerdings auch Gefahren verbunden - vor allem, wenn die Wärmeregulation durch Erkrankungen oder Medikationen auf zentraler oder lokaler Ebene gestört ist. Dies geschieht vor allem in Hinblick auf die Herzkreislaufbelastung und auf thermische Schädigungen der Haut. Daher sind Wärmeapplikationssysteme zu fordern, die den Wärmefluss individuell an die Wärmeaufnahmefähigkeit des Körpers anpassen. Als Regelgrössekann dazu diekontinuierliche Messung der Hauteigentemperatur genutzt werden, um eine effiziente und sichere Wärmeanwendung zu ermöglichen.

\section{KARGER}

Fax +497614520714 Information@Karger.com www.karger.com

\section{Keywords}

Thermoregulation - Thermoneutral zone · Sauna · Infrared cabin . Infrared rays - Skin temperature - Skin temperature measurement . Erythema ab igne $\cdot$ Burn injury

\section{Summary}

Thermal Therapies, Taking into Account the Human Thermoregulatory System-Part 1

Thermal therapies in health promotion and alleviation of various diseases are an essential part of our culture. However, advances in medicine have pushed thermal therapies as a therapeutic method to the periphery. The demographic change, the increase of chronic and metabolic diseases, and the rising costs in healthcare are leading once again to a growing interest among both patients and experts in medicine regarding thermal therapies. Beside conduction and convection, the radiative transfer of thermal energy is widely used both in the private and in the medical environment. The effects of heat application, however, are independent from their source. Heat application leads to reflexive reactions (e.g. muscle relaxation and pain relief) as well as effects based on the increasing blood flow and the temperature in the tissue. As heat can only be distributed in the body by convection via the blood, effects on the cardiovascular system can be easily explained. Regarding the human thermoregulatory system, three principles of heat application can be distinguished. Heat application to a human body can be applied in an environment above, within, or below the thermal neutral zone. In each of those cases, both the heat distribution and the resulting cardiovascular burden differ, especially since diseases or medication affect the thermoregulatory system at a central or local level. This may result in a higher risk of cardiovascular burden and thermal damage to the skin. As a result, heat delivery systems should allow an adjustment of the heat flux to the individual heat capacity of the skin and body. As a control variable regarding this aspect, a continuous measurement of skin temperature provides the necessary information to establish an effective and safe thermal therapy. 


\section{Mots clés}

Thermorégulation · Zone thermoneutre · Sauna · Cabine infrarouge · Rayonnement infrarouge $\cdot$ Température corporelle $\cdot$ Mesure de la température corporelle $\cdot$ Érythème ab igne $\cdot$ Brûlure

\section{Résumé}

Applications de chaleur avec régulation thermique - Partie 1

Les applications de chaleur utilisées dans la prévention des maladies et pour soulager diverses douleurs font partie intégrante de notre culture. Avec les progrès de la médecine, les applications de chaleur en tant que procédé thérapeutique ont été tout simplement oubliées. Avec l'évolution démographique, l'augmentation des maladies chroniques et surtout métaboliques, mais aussi en raison de la pression des coûts du système de santé, l'intérêt pour ce procédé croît auprès des personnes concernées et dans la médecine également. En plus de la conduction et de la convection, des procédés de rayonnement sont utilisés dans le milieu privé et médical. Les effets de l'application de chaleur peuvent s'expliquer indépendamment du support avec réactions réfléchissantes (détente des muscles et soulagement de la douleur) et des effets basés sur l'augmentation de la circulation sanguine et de la température dans les tissus. La chaleur dans le corps peut être diffusée dans le sang presque exclusivement par convection, d'où l'influence sur le système cardiovasculaire. En prenant en considération les mécanismes de régulation thermique, on distingue trois principes. L'application de chaleur au-dessus, à l'intérieur et en-dessous de la zone thermoneutre est différente en ce qui concerne la charge du système cardiovasculaire et la diffusion de la chaleur. Les applications de chaleur représentent toutefois des risques également, surtout lorsque la régulation thermique est perturbée sur le plan central ou local par des maladies ou des médicaments. Cela se produit surtout compte tenu de la charge du système cardiovasculaire et des dommages thermiques sur la peau. Par conséquent, il faut exiger des systèmes d'application de chaleur qui adaptent le flux de chaleur individuellement à la capacité d'absorption de chaleur du corps. La mesure continue de la température propre de la peau peut être utilisée en tant que grandeur réglée pour permettre une application de chaleur efficace et sûre.

\section{Einleitung}

Die Erkenntnis, dass Wärme zur Linderung unterschiedlichster Beschwerden eingesetzt werden kann, ist vermutlich so alt wie der Mensch selbst. Praktisch jede Hochkultur ersann zahllose Methoden, dem menschlichen Organismus lokal und/oder systemisch Wärme zuzuführen.

Die Fortschritte der Medizin im letzten Jahrhundert verdrängten dieses Wissen weitgehend in die Randgebiete. Heute gewinnen sie jedoch wieder zunehmend an Bedeutung, was letztlich auf die demographische Entwicklung der Gesellschaft und die veränderten Lebensumstände zurückzuführen ist. Chronische, vor allem gefäss- und stoffwechselbedingte Erkrankungen nehmen massiv zu. Die Kosten im Gesundheitswesen explodieren und den Menschen wird immer mehr Eigenverantwortung in der Gesundheitsvorsorge und Gesunderhaltung abverlangt.

Unterstützt durch die Erkenntnis «Alle wollen alt werden, aber keiner will alt sein», hat sich ein breites Angebot an Spa-, Wellness- und Heimanwendungen entwickelt. Viele dieser Anwendungen, die letztlich nur auf eine Wärmewirkung zurückzuführen sind, werden mit einem Postulat nichtthermischer Wirkungen verbunden, das selten durch klinische Daten belegt werden kann. Der Blick auf das Wesentliche - Wärme als unspezifisches, aber effektives Wirkprinzip - tritt hierbei immer mehr in den Hintergrund. Dabei lassen sich die Effekte von Wärmeanwendungen hinreichend durch zwei Prinzipien erklären: die Auswirkungen durch eine Temperaturerhöhung im Gewebe und durch die Anregung der Wärmeregulationsmechanismen.

Das komplexe Wärmeregulationssystem, das die Homöothermie ermöglicht, ist gut untersucht. Dennoch finden sich kaum Überlegungen, dieses Wissen zu nutzen, um dem Körper möglichst belastungsfrei Wärme zuzuführen. Die «Wärmeapplikation» steht dem Bestreben des Organismus nach Homöothermie entgegen.

Wärmeapplikationen setzen zunächst die Haut einer thermischen Belastung aus - vor allem, wenn die Wärmeregulation beeinträchtigt ist. Die Wärmeabwehrreaktionen werden stufenweise ausgelöst und führen in erster Linie zu Belastungen des Herzkreislaufsystems.

Die folgende Übersicht soll unterschiedliche Wärmeanwendungen unter Berücksichtigung der Thermoregulationsfähigkeit des menschlichen Körpers beleuchten. Daraus sind mögliche Grundprinzipien über Effekte von Wärmeapplikationen ableitbar. Die physiologischen Grundlagen der Wärmeregulation/Wärmeabwehrreaktion zum Schutz der Haut und zur Konstanz der Körperkerntemperatur sollten dabei berücksichtigt werden. Wärmeanwendungen lassen sich so gezielter, effizienter und vor allem sicherer gestalten.

\section{Grundlagen der Wärmeregulation}

\section{Definition der Wärme}

Die Physik trennt die Begriffe «Wärme», thermische Energie und Temperatur. Umgangssprachlich verwischt diese Trennung, sodass der Begriff «Wärme» aus physikalischer Sicht oft inkorrekt verwendet wird.

In der Thermodynamik ist Wärme auch über Systemgrenzen hinweg transportierte thermische Energie. Wärme ist also wie Arbeit an Transportvorgänge gebunden und somit eine Prozessgrösse. Entsprechend des zweiten 

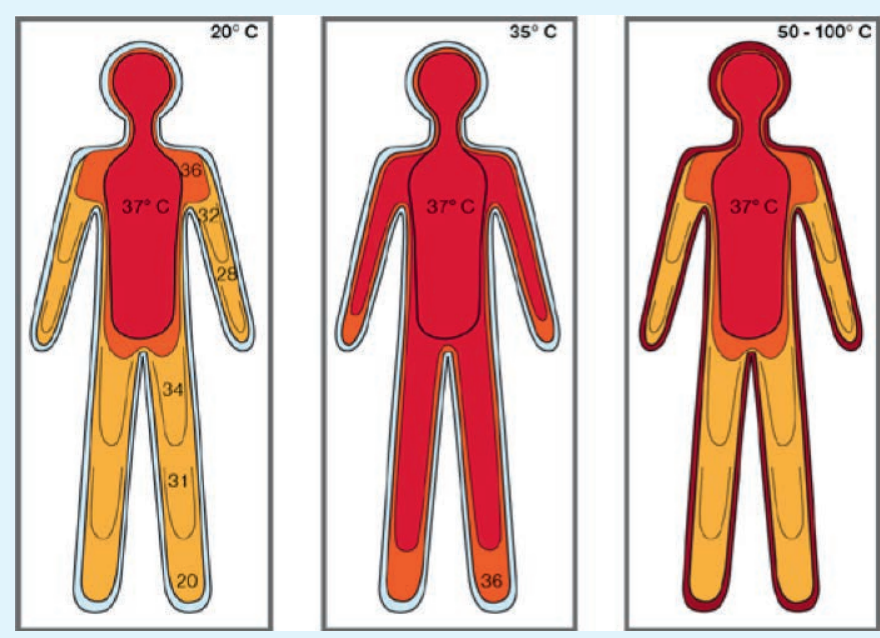

Hauptsatzes der Thermodynamik wird zwischen thermisch gekoppelten Systemen Wärme immer von Systemen mit höherer Temperatur auf Systeme mit niedrigerer Temperatur übertragen. Dies gilt, bis zwischen den Systemen ein thermisches Gleichgewicht besteht.

Wird «thermische Energie» übertragen, so führt dies nicht zwangsläufig zu einer Temperaturerhöhung, sondern vielmehr zu einer Zustandsänderung. Die Wärmezufuhr kann zu einer Temperaturerhöhung, einem Phasenübergang (z.B. das Schmelzen von Eis) oder einer Druckänderung (bei einem Gas) führen.

Auf molekularer Ebene kann man thermische Energie als ungerichtete Schwingung der Materie, d.h. als Vibration von Atomen bzw. Molekülen, verstehen (Brown'sche Molekularbewegung). Die Schwingungsenergie verhält sich dabei proportional zur Temperatur der Materie.

Grundsätzlich stehen vier physikalische Prinzipien für den Transport thermischer Energien zur Verfügung:

- Konduktion: der Wärmetransport in/zwischen festen Körpern durch die Brown'sche Molekularbewegung,

- Konvektion: der Wärmetransport durch ein bewegtes Medium (Gase oder Flüssigkeiten),

- Radiation: Wärmetransport durch elektromagnetische Strahlung,

- Evaporation: der Wärmeverlust durch Verdunstung.

\section{Temperaturabhängigkeit des Stoffwechsels}

Die Stoffwechselprozesse lebender Organismen sind stets mit einer Wärmeproduktion verbunden und folgen thermodynamischen Gesetzmässigkeiten. Die Stoffwechselrate ist dabei selbst temperaturabhängig. Die Reaktions-Geschwindigkeits-Temperatur-Regel (RGT-Regel) von van't Hoff beschreibt diesen Zusammenhang für biochemische Prozesse:

$$
\mathrm{Q} 10=\mathrm{k}_{(\mathrm{T}+10)} / \mathrm{k}_{(\mathrm{T})}
$$

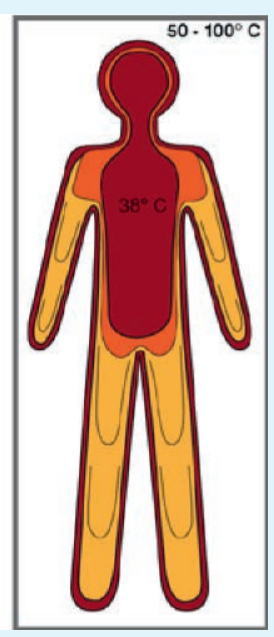

Abb. 1. Isothermen und KKT (modifiziert nach $[1,4]$ ).
Der Quotient Q10 gibt das Verhältnis zwischen den Reaktionsgeschwindigkeiten $(\mathrm{k})$ bei $10^{\circ} \mathrm{C}$ Temperaturdifferenz an. Für biologische Systeme sind Faktoren von 2-3 typisch. Wird im Gewebe die Temperatur um $10^{\circ} \mathrm{C}$ angehoben, steigt der Energieverbrauch (P) um den Faktor 2-3:

$$
\mathrm{P}_{1} / \mathrm{P}_{2}=\mathrm{Q} 10^{(\mathrm{T} 1-\mathrm{T} 2) / 10}
$$

Auch die Diffusion im Gewebe nimmt bei einer Temperaturerhöhung um $10{ }^{\circ} \mathrm{C}$ um etwa $3 \% \mathrm{zu}[1,2]$.

\section{Homöothermie}

Temperaturveränderungen beeinflussen direkt die Geschwindigkeit vor allem biochemischer Reaktionen. Evolutionsbiologisch bietet die Homöothermie Vorteile, da hinsichtlich des Lebensraums eine grössere Unabhängigkeit von Umweltbedingungen möglich wird. Säugetiere und Vögel haben einen hohen Ruheenergieumsatz mit hoher Wärmebildungsrate. Ihre Körpertemperatur liegt in der Regel über der Temperatur der Umgebung.

Um temperaturbedingte Änderungen der Leistungsfähigkeit zu vermeiden, wird die Körpertemperatur im Inneren weitestgehend konstant gehalten. Aus physiologischer Sicht ist die autonome Steuerung der Körpertemperatur ein zentraler Aspekt der Homöostase. Die Körperkerntemperatur ist dementsprechend «scharf» eingestellt und stellt eine hierarchisch übergeordnete Regelgrösse dar $[2,3]$.

\section{Konstanz der Körpertemperatur - Körperkern, Körperschale, äussere Körperschale}

Die Konstanz der Körpertemperatur betrifft nur den Körperkern (Körperkerntemperatur, KKT) mit den inneren Organen und dem Zentralnervensystem. Die Tem- 
peratur der Peripherie oder «Körperschale» (Muskulatur, Binde- und Fettgewebe, Knochen und Gelenke) ist grösseren Schwankungen unterworfen. Sie ist von der Umgebungstemperatur und der Wärmebildung abhängig. Die Bereiche gleicher Temperatur bezeichnet man als Isothermen. Für die Verschiebung der Isothermen in der Körperschale ist die wechselnde Durchblutung der einzelnen Körperpartien verantwortlich. Sie ist eine wichtige temperaturregulatorische Massnahme in beide Richtungen - sowohl gegen Unterkühlung als auch gegen Überhitzung. Die Körperschale erfüllt damit gewissermassen die Funktion eines Wärmepufferspeichers (Abb. 1, 2).

Die normale KKT wird mit ca. $37^{\circ} \mathrm{C}$ angegeben. Sie ist individuellen und physiologischen Schwankungen unterworfen. Die Messung der tatsächlichen KKT ist nicht trivial.

Sublinguale und axillare Messungen sind fehlerhaft und geben zu niedrige Werte an. Die Messung der Trommelfelltemperatur erweist sich in der Praxis als unzuverlässig (korrekte Einstellung des Trommelfells, Cerumen usw.). Eine gute Korrelation weist die rektale Messung auf. Sie ist in der ärztlichen Praxis gut umsetzbar, hat eine relativ geringe Fehlerquote und ist nach wie vor Standard. Zum kontinuierlichen Monitoring in der Intensivmedizin haben sich die Ösophagussonde sowie die Messung der

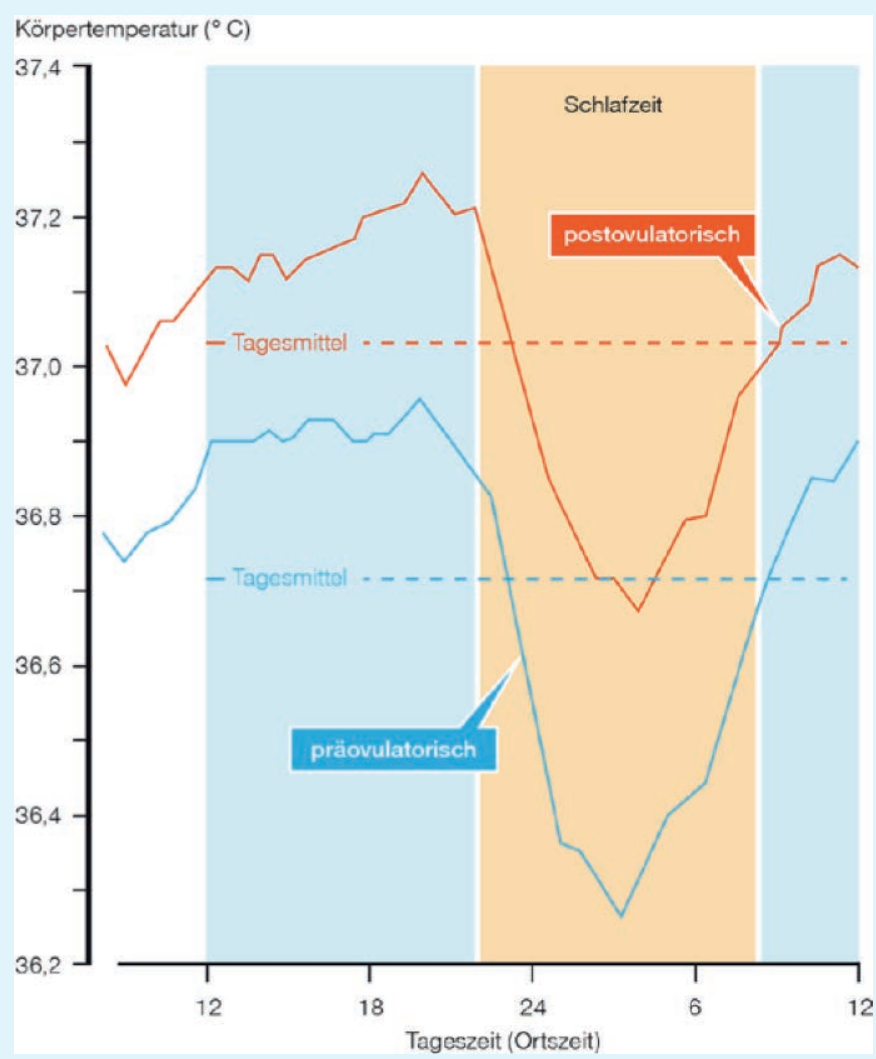

Abb. 2. Körpertemperaturen zu den verschiedenen Tageszeiten (modifiziert nach $[1,4])$.
Tab. 1. Überblick über die Körperkerntemperaturen und deren physiologische Konsequenzen (modifiziert nach $[1,8]$ )

\begin{tabular}{ll}
\hline KKT, ${ }^{\circ} \mathrm{C}$ & Physiologische Konsequenzen \\
\hline$<20$ & Asystolie, «Kältetod» \\
$20-27$ & «Scheintod», lichtstarre Pupillen, extreme Bradykardie \\
$27-30$ & tiefe Hypothermie, Versagen der Thermoregulation, \\
& Kammerflimmern \\
$30-33$ & Stoffwechselsenkung, Atemdepression, Bewusstseinstrübung \\
$33-36$ & milde Hypothermie, Kältezittern, Stoffwechselsteigerung \\
$36-38$ & Normothermie \\
$38-40$ & Fieber, Hyperthermie \\
$40-44$ & Versagen der Thermoregulation, Hitzschlag, Krämpfe \\
$>44$ & Denaturierung von Eiweissen, «Hitzetod» \\
\hline
\end{tabular}

Kerntemperatur mittels Rechtsherzkatheter (Swan-GanzKatheter) durchgesetzt. Eine aktuelle Entwicklung zur nichtinvasiven Messung der KKT ist der sogenannte Doppelsensor. Dieser wird an der Stirn platziert und korreliert sehr gut mit der rektalen Temperaturmessung [5-7].

Tabelle 1 gibt einen Überblick über die KKTs und deren physiologische Konsequenzen. Die Angaben hierzu differieren in der Literatur. Offensichtlich sind KKTs bis knapp unter $14{ }^{\circ} \mathrm{C}$ mit dem Leben vereinbar. Allerdings ist es die Summe verschiedenster Umstände, die letztendlich die Überlebensprognose bestimmen, wie unter anderem Unterkühlungsform (Lawine, BeinaheErtrinken), Geschwindigkeit des Auskühlens, Möglichkeiten der Reanimationsmassnahmen und Nähe des nächstgelegenen medizinischen Versorgungszentrums $[1,9]$.

\section{Thermoregulation}

Eine relative Konstanz der KKT erfordert, dass aktivitätsbedingte Schwankungen der Thermogenese (eigene Wärmeproduktion) und wechselnde thermische Umgebungsbedingungen ausgeglichen werden müssen.

Die stetige Balance zwischen der Thermogenese sowie der Wärmeabgabe an die und der Wärmeaufnahme aus der Umwelt erfordert eine effiziente Thermoregulation. Diese wird durch das Zusammenspiel komplexer und untereinander vernetzter Regelkreise, jeweils bestehend aus Regulationszentrum, Rezeptor und Effektor, realisiert.

Die Regelzentren sind auf mehreren Ebenen des Zentralnervensystems angelegt (Hypothalamus, Mesencephalon, Rückenmark). Die Fühler der Thermoregulation sind unterteilt in innere und äussere Sensoren. Als äussere Sensoren fungieren die Thermorezeptoren der Haut. Die Kältesensoren weisen eine maximale Empfindlichkeit bei 


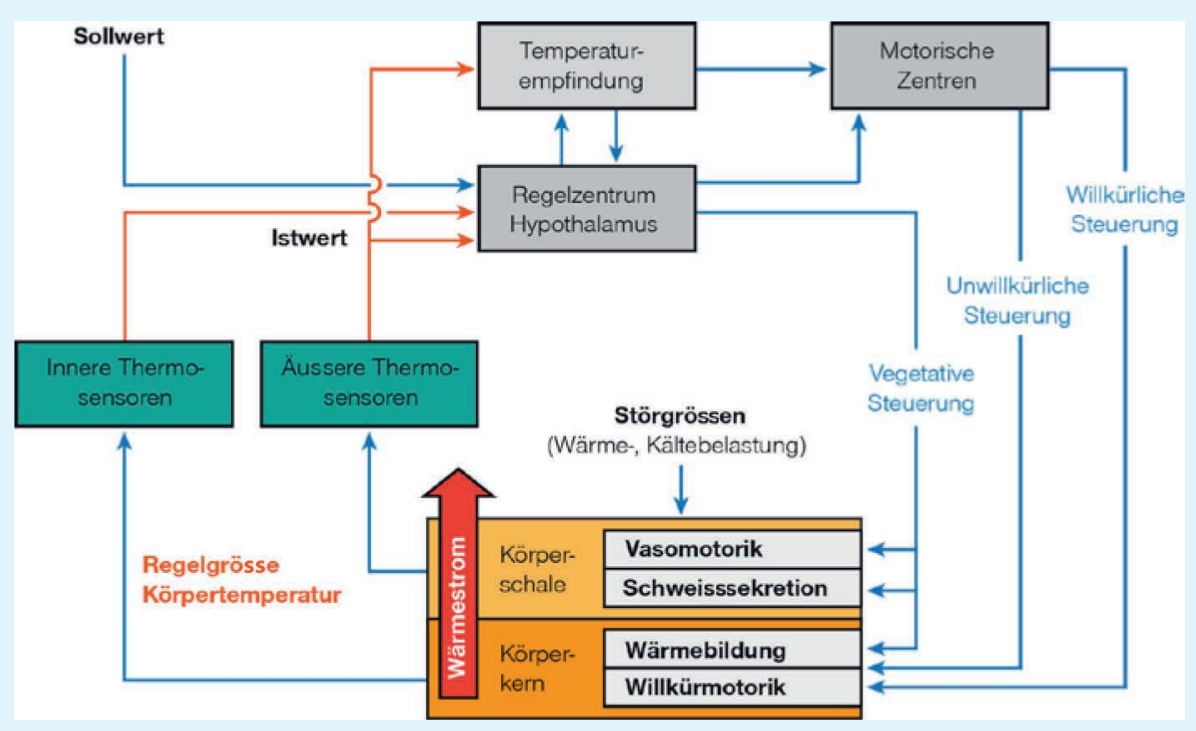

Abb. 3. Wärmeregulation (modifiziert nach [4]).

ca. $30^{\circ} \mathrm{C}$ auf, die Wärmesensoren bei ca. $43^{\circ} \mathrm{C}$. Im Körperinneren finden sich thermosensible Strukturen im Hypothalamus, im Rückenmark sowie in der Bauchhöhle und im Thorax $[2,4,10]$.

Vegetative Effektoren:

- Hautgefässe mit sympathischer, vasokonstriktorischer Innervation.

- Intestinale Gefässe mit sympathischer, vasokonstriktorischer Innervation.

- Schweissdrüsen mit cholinerger, sympathischer Innervation.

- Sympathische Innervation der Leber, der Muskulatur, des Fettgewebes und des Nebennierenmarks (Steigerung des Stoffwechsels).

- Schilddrüse.

Somatomotorische Effektoren:

- Skelettmuskulatur einschliesslich der Atemmuskulatur und thermoregulatorischer Verhaltensweisen wie Körperhaltung, Kältezittern, Wärmehyperpnoe und Hechelatmung.

Ein wesentliches Merkmal der Thermoregulation ist, dass die verfügbaren Mechanismen je nach Umgebungsbedingung einzeln oder in unterschiedlicher Kombination eingesetzt werden können (Abb. 3).

Nur wenn Wärmebildung, Wärmeabgabe (Summe aus Konduktion, Konvektion, Abstrahlung, Evaporation) und Wärmeaufnahme (Summe aus Konduktion, Konvektion, Strahlung) ausgeglichen sind, kann die KKT konstant gehalten werden. Eine Nettowärmeaufnahme führt zwangsläufig zu einem Temperaturanstieg und ist nur äusserst begrenzt tolerierbar.

\section{Wärmebildung und innerer Wärmetransport}

\section{Thermogenese (Wärmebildung)}

Die Wärmebildung im menschlichen Organismus ist eine Funktion des Energieumsatzes mit einem Wirkungsgrad $\leq 25 \%$. Sie wird unter anderem durch die Nahrungsaufnahme, die Muskelaktivität, die Herz- und Atemfunktion sowie die Sekretion verschiedener Hormone (Adrenalin, Thyroxin usw.) beeinflusst. In Ruhe beträgt die Wärmeproduktion bei einem $70 \mathrm{~kg}$ schweren Erwachsenen etwa 80 Watt; d.h., auch in Ruhe wird ständig Wärme an die Umwelt abgegeben $[1,11]$.

Körperliche Aktivität erhöht die Körperschalentemperatur (KST) und die KKT. In Ruhe werden ca. 16\% der Wärme vom Gehirn, 56\% von den Brust- und Bauchorganen sowie $18 \%$ von Haut und Muskeln generiert. Bei körperlicher Arbeit verschieben sich die Verhältnisse dramatisch, sodass $90 \%$ der Wärme von der Muskulatur produziert werden. Während eines Marathonlaufs kann durchaus eine physiologische KKT von $39{ }^{\circ} \mathrm{C}$ erreicht werden [12].

Wie bei der normalen Muskelarbeit entsteht auch durch Kältezittern Wärme. Beim Zittern wird sie zur Thermogenese verwendet. Beim Mensch beträgt die Ausbeute aber nur ca. $11 \%$. Eine «zitterfreie Thermogenese» oder auch "chemische Thermogenese» ermöglicht das «braune Fettgewebe». Es findet sich nur bei Säuglingen und ist auf bestimmte Körperregionen beschränkt $[3,13]$.

\section{Innerer Wärmetransport}

Unter Ruhebedingungen ist die Temperatur im Körperkern höher als in der Peripherie. Es ist ein stetiger «innerer Wärmetransport» von innen nach aussen erfor- 
Tab. 2. Wärmeleitzahlen [14]

\begin{tabular}{ll}
\hline Feststoff & Wärmeleitzahl Y, W/(m $\times \mathrm{K})$ \\
\hline Fettgewebe, stark durchblutet & 0,4 \\
Fettgewebe, wenig durchblutet & 0,2 \\
Epidermis & 0,3 \\
Luft & 0,03 \\
Wasser & 0,6 \\
Vakuum & 0,4 \\
Aluminium & 200 \\
Stahl & 60 \\
Stahlbeton & 2,3 \\
Hartholz & 0,2 \\
Mineralwolle/Styropor & 0,04 \\
\hline
\end{tabular}

derlich, um die Konstanz der KKT zu gewährleisten. Für den «inneren Wärmetransport» kommen die Konduktion und die Konvektion infrage.

\section{Konduktion}

Der Wärmezufluss wird durch die Temperaturdifferenz und die Wärmeleitzahl bestimmt $[4,14]$. So wird $60{ }^{\circ} \mathrm{C}$ heisses Styropor (Wärmeleitzahl 0,04) bei Kontakt mit der Haut keine Verbrennung verursachen, $60^{\circ} \mathrm{C}$ heisses Aluminium (Wärmeleitzahl 200) dagegen schon - immer vorausgesetzt, dass die normale Wärmeabwehrreaktion der Haut erhalten ist.

Für die Effektivität der Konduktion ist die Wärmeleitfähigkeit eines Stoffs entscheidend. Diese wird durch die Wärmeleitzahl Y beschrieben (Tab. 2).

Das Körpergewebe weist keine hohe Wärmeleitfähigkeit auf. Die Konduktion kommt daher in erster Linie bei der Wärmeabgabe aus den Hautblutgefässen zur äusseren Haut zum Tragen. Für den Wärmefluss ist neben der effektiven Diffusionsfläche die Temperaturdifferenz zwischen Blutgefäss und Hautoberfläche sowie der Abstand des Gefässes von der Hautoberfläche massgebend $[1,13,14]$.

\section{Konvektion}

Der «innere Wärmetransport» erfolgt somit fast ausschliesslich durch Konvektion mithilfe des fliessenden Blutes. Die Grösse des Wärmestroms hängt von der Durchblutung der Körperschale bzw. Haut ab. Diesen Zusammenhang veranschaulicht die Wärmedurchgangszahl (U-Wert), die in $\mathrm{W} / \mathrm{m}^{2} \mathrm{~K}$ angegeben wird.

Der U-Wert von Baustoffen liegt zum Vergleich im Bereich von 0,3 (Mauerziegelaussenwand mit Wärmedämmung, $30 \mathrm{~cm}$ ), 0,5 (Massivholzaussenwand, 20,5 cm), 1,5 (Mauerziegelaussenwand $24 \mathrm{~cm}$ ) und 3,3 (Betonaussenwand $25 \mathrm{~cm}$ ).

Für die Strecke vom Körperkern zur Hautoberfläche wird ein U-Wert-Bereich von ca. 7-50 angegeben. Allein die Vasokonstriktion der peripheren Gefässe kann den U-Wert dabei um 50\% variieren [2].
Zur Minderung von Wärmeverlusten in der Peripherie dient das Gegenstromprinzip. Abgekühltes venöses Blut aus der Peripherie entzieht dem arteriellen Blut Wärme, sodass das arterielle Blut bereits abgekühlt ist, bevor es die Kapillaren der Haut erreicht. Das Blut in der Arteria radialis kann so bis zu $8{ }^{\circ} \mathrm{C}$ kälter sein als das in der Arteria brachialis. Ferner kann Blut, bevor es die Kapillaren der Haut erreicht, über arteriovenöse Anastomosen zu einem grossen Teil wieder direkt zurückgeführt werden.

Das Zirkulationssystem der Körperschale, AV-Shunts und Gegenstromprinzip, mindert die Wärmeverluste in der Peripherie. Es kann aber auch dazu genutzt werden, den Anstieg der Körperkerntemperatur in heissen Umgebungen hinauszuzögern, was im Umfeld von Wärmeanwendungen zu berücksichtigen ist.

Insgesamt gesehen ist also der konvektive Wärmetransport über das Blut der quantitativ entscheidende Mechanismus. Die Umverteilung des Blutes als aktive und zielgerichtete Leistung spielt daher die zentrale Rolle bei den möglichen Wärmeregulationsreaktionen [1,2].

\section{Wärmeabgabe an die Umwelt - äusserer Wärmetransport}

\section{Äussere Körperschale - Haut und Schleimhaut}

Die Wärmeabgabe an die Umwelt erfolgt über die äussere Körperschale (Haut) und die Atemwege. Neben Konduktion und Konvektion kommen auch die Wärmeabstrahlung (Radiation im Infrarot-C-Bereich) und die Verdunstung (Evaporation) zum Tragen. Konduktion, Konvektion und Radiation fasst man auch unter dem Begriff der «trockenen Wärmeabgabe» zusammen.

Die Wärmeabgabe über die Atemwege hat einen Anteil von ca. $10 \%$. Dem Körper wird Wärme entzogen, da die Inspirationsluft angewärmt und mit Feuchtigkeit gesättigt werden muss. 90\% der Wärmeabgabe erfolgt über die Haut (Körperschale) (Tab. 3).

Tab. 3. Prozentuale Verteilung der Wärmeabgabe bei Indifferenztemperatur, $50 \%$ relativer Luftfeuchtigkeit und Windstille [4]

\section{Haut \\ Abstrahlung (IR-C) 45\% \\ Konduktion und Konvektion 25\% \\ Wasserverdunstung $20 \%$}

\section{Gesamt: $90 \%$}

\section{Atemwege}

Konduktion und Konvektion 2\%

Wasserverdunstung 8\%

Gesamt: $10 \%$ 


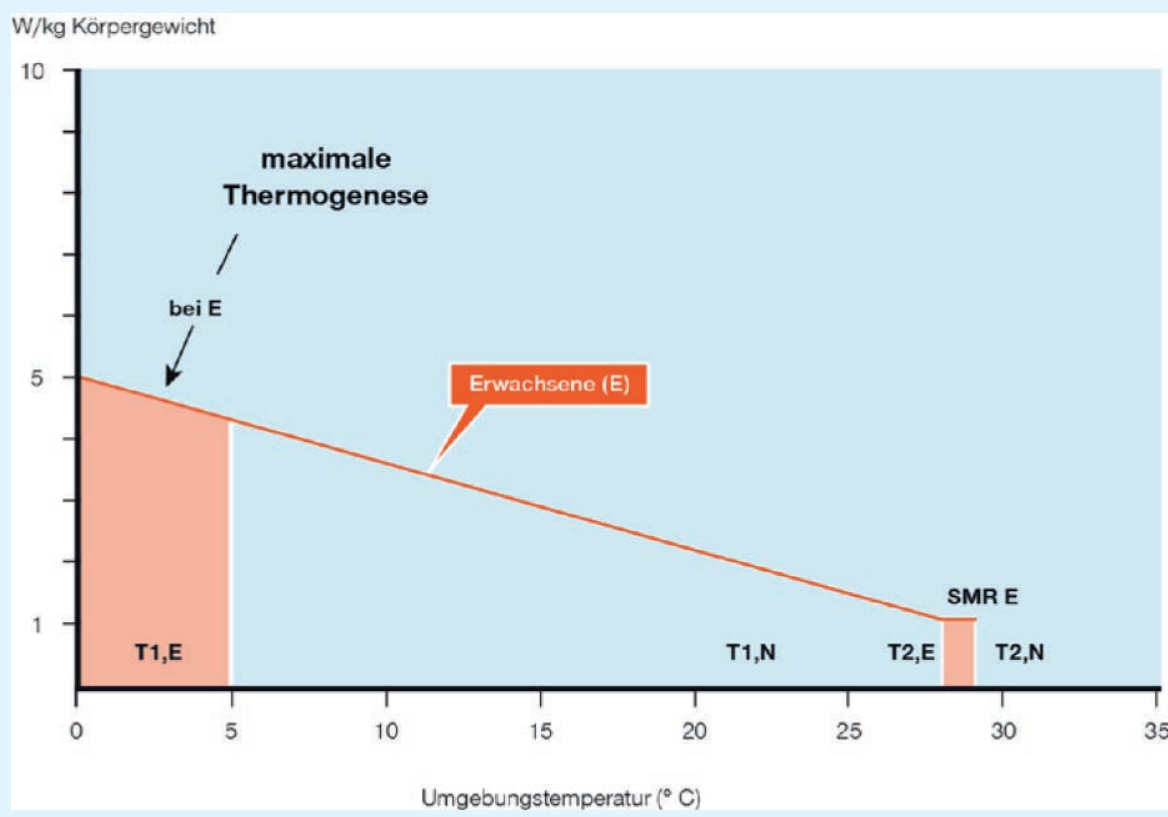

Abb. 4. Thermogenese (modifiziert nach [15]).

Körperoberfläche, Kinder und ältere Menschen

Mit zunehmender Körpergrösse bei gleichen relativen Körpermassen steigt die Oberfläche im Quadrat und die Körpermasse im Kubik. Bei kleinen Personen, vor allem aber bei Säuglingen und Kleinkindern, ist daher das Verhältnis von Wärme verlierender oder Wärme aufnehmender Hautoberfläche zu Wärme generierender Körpermasse gross. Diese Personengruppe ist dementsprechend eher gefährdet, auszukühlen oder zu überhitzen [2].

Bei älteren Menschen ist zudem zu berücksichtigen, dass häufig eine Einschränkung der Herzkreislauffunktion besteht, Gefässveränderungen vorliegen und nicht selten die Flüssigkeitszufuhr zu niedrig ist. Dies sind Faktoren, die die Wärmeregulationsfähigkeit beeinträchtigen und schnell zu hohen Belastungen führen (Abb. 4).

\section{Transepidermaler Wasserverlust - gesamter täglicher Wasserverlust}

Der Körper gibt über die Haut und Schleimhäute ständig Wasser an die Umgebung ab. Dieser Wasserverlust erfolgt passiv durch Diffusion, ohne aktive, ekkrine Beteiligung (Perspiratio insensibilis), und beträgt ca. 0,375 1 [2].

Der tägliche Wasserverlust eines Menschen addiert sich so zu ca. 2,5 1 unter Ruhebedingungen, bei $20^{\circ} \mathrm{C}$ Lufttemperatur und bei einer Luftfeuchtigkeit von 40-60\% (Urin 1,5 l, Kot 0,15 l, Atemluft 0,375 l, Haut 0,375 l, Schweiss $0,1 \mathrm{l})$.

\section{Wärmeabgabemöglichkeiten}

\section{Konduktion}

Unter Konduktion versteht man den Wärmetransport in festen Körpern über die Brown'sche Molekularbewegung (Wärmediffusion, Wärmeleitung). Die Wärmeabgabe durch Konduktion kann durch direkten Kontakt mit einer kälteren Oberfläche erfolgen. Der Wärmefluss wird dann durch die Temperaturdifferenz, die Wärmeleitfähigkeit des Stoffes und die Grösse der Kontaktfläche bestimmt.

Unter normalen Bedingungen entsteht jedoch am Übergang von der Hautoberfläche zur Umgebungsluft eine stehende Luftschicht. Sie bildet eine Grenzschicht mit guten Isolationseigenschaften; entsprechend geringer ist die Wärmeleitung. Der Wärmefluss hängt von der Temperaturdifferenz zwischen Haut und umgebender Luft sowie der Dicke der Grenzschicht über der Haut ab.

\section{Konvektion}

Die Konvektion beschreibt den Wärmetransport durch bewegte Medien (Gase oder Flüssigkeiten).

Über der Haut bildet sich eine stehende Luftschicht. Sie dient als Isolation und verringert den Wärmeverlust. Ausserhalb dieser Luftschicht wird der Wärmetransport durch Konvektion mithilfe von zirkulierender Luft ermöglicht. Diese gewährleistet einen deutlich höheren Wärmefluss.

Die Grenzschichtdicke nimmt bei Luftbewegungen (Wind, Ventilator) massiv ab (und damit die Wärme- 
abgabe zu). Die Wärmeabgabe ist proportional der Wurzel der Strömungsgeschwindigkeit. Bei starkem Wind steigert die erhöhte Konvektion die Wärmeabgabe und erniedrigt die Hauttemperatur. Die strömende Luft wird bei gleicher Temperatur als kälter empfunden. Dieser als Windchill-Faktor bezeichnete Effekt lässt sich nach einer vereinfachten Formel ( $\mathrm{T}=$ Umgebungstemperatur; $\mathrm{V}=$ Windgeschwindigkeit in $\mathrm{km} / \mathrm{h}$; SQRT $=$ Quadratwurzel) bestimmen:

$$
\mathrm{TWC}=33+(0,487+0,237 \mathrm{SQRT}(\mathrm{V})-0,0124 \mathrm{~V}) \times(\mathrm{T}-33)
$$

Bei einer Lufttemperatur von $-8{ }^{\circ} \mathrm{C}$ und einer Windgeschwindigkeit von 4 Beaufort (im Mittel $24 \mathrm{~km} / \mathrm{h}$ ) errechnet sich beispielsweise eine gefühlte Temperatur von $-22^{\circ} \mathrm{C}[2]$.

Im Wasser gilt Entsprechendes. Es bildet sich eine Grenzschicht zwischen Hautoberfläche und Wasser. Die Dicke ist aber im Vergleich zur Luft auf 1/10 reduziert. Wasser hat zudem eine 20 -fach höhere Wärmeleitfähigkeit als Luft. Ein Schwimmer ist daher einer 200-fach höheren Wärmeabgabe ausgesetzt.

\section{Radiation - Infrarotstrahlung}

Nach dem Planck'schen Strahlungsgesetz gibt jede Oberfläche ein Spektrum elektromagnetischer Strahlung $a b$, das durch die Oberflächentemperatur definiert ist.

Die Bilanz von Wärmeabgabe und -aufnahme durch Infrarotstrahlung ist eine Funktion der Hauttemperatur sowie der Temperatur Infrarot-abstrahlender Körper (Wände, Gegenstände und Körper im Raum, Sonne). Zu berücksichtigen ist, dass kurzwellige Infrarotstrahlung zu 50\% durch die Hautpigmente reflektiert wird (der Anteil trägt nicht zur Erwärmung der Haut bei), während langwellige Infrarotstrahlung von der Haut nahezu vollständig absorbiert wird [16].

Die Bilanz des Wärmeaustauschs über Infrarotstrahlung wird bei kleinen Temperaturgradienten näherungsweise mithilfe des Stefan-Bolzmann-Gesetzes errechnet:

$$
\operatorname{Hrad}=\mathrm{k}^{\star} \text { Aeff }{ }^{\star}(\text { THaut }- \text { TWand })
$$

\section{Evaporation - Verdunstung}

Die Evaporation beschreibt den Wasserverlust des Körpers über die Haut und die Schleimhäute durch Schweissbildung bzw. Wasserdampf. Im Gegensatz zur Perspiratio insensibilis ist die Schweisssekretion (Perspiratio sensibilis) ein aktiver, vegetativ-nerval gesteuerter Prozess. Die Schweissdrüsen werden durch thermische, körperliche oder emotionale Reize aktiviert (glanduläre

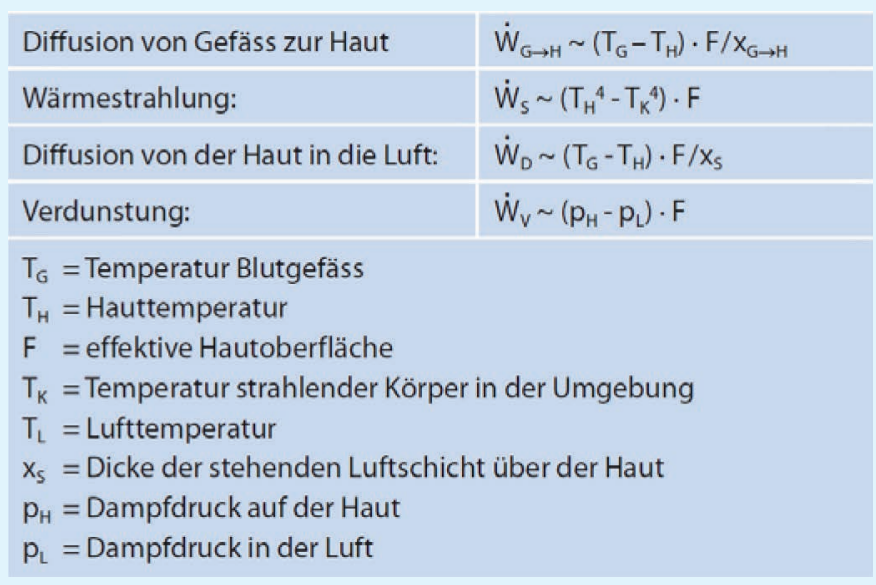

Abb. 5. Wärmetransport in der Haut (modifiziert nach [4]).

Evaporation). Die Verdunstung von Wasser benötigt 2400 kJ/l. Das Verdunsten von 0,5 1 Wasser entzieht dem Körper ein Sechstel der Ruhewärmeproduktion eines Tages. Die Schweissbildung ist daher ein sehr starker Effektor der Thermoregulation [2, 4].

Diese Form des gerichteten Transports thermischer Energie nutzt der Körper zur Abgabe überschüssiger Wärme an die Umwelt. Die abgegebene Wärmemenge hängt vom Verhältnis des Wasserdampfdrucks $(\mathrm{P})$ über der Hautoberfläche und der Luft ab. Der Dampfdruck hängt wiederum von der Temperatur und der Feuchtigkeit der Hautoberfläche bzw. der Luft ab:

$$
\mathrm{H}_{\text {Evaporation }}=\text { alpha }{ }^{\star} \text { Aeff } *\left(\mathrm{P} \mathrm{H}_{2} \mathrm{O} \text { Haut }-\mathrm{P} \mathrm{H}_{2} \mathrm{O} \text { Umgebung }\right)
$$

Wärme kann durch Verdunstung nur dann abgegeben werden, wenn der Wasserdampfdruck der Haut grösser ist als derjenige der Luft. Selbst bei vollständig wasserdampfgesättigter Luft ist das noch möglich, wenn die Hauttemperatur höher ist als die Temperatur der umgebenden Luft. Ist die Umgebungstemperatur höher als die Körperschalentemperatur, bleibt dem Organismus nur die Wärmeabgabe über Verdunstung. Mit steigender Luftfeuchtigkeit vermindert sich der Wärmefluss. Ist die Luft wasserdampfgesättigt, versagt auch die Möglichkeit der evaporativen Wärmeabgabe (z.B. in den Tropen) (Abb. 5).

\section{Thermoregulation bei unterschiedlichen Umgebungsbedingungen}

Je nach Umgebungsbedingung werden vegetative und somatomotorische Effektoren einzeln oder in Kombination eingesetzt, um die KKT konstant zu halten. Die jeweilige Einstellung der Mechanismen zur Wärme- und Kälteabwehr erfolgt zentral auf Basis der Informationen der peripheren und zentralen Thermosensoren. 


\section{Thermoneutralzone und Indifferenztemperatur}

Kann allein durch Vasodilatation oder Vasokonstriktion der Hautblutgefässe eine Balance zwischen Wärmeproduktion und Wärmeabgabe bzw. Wärmeaufnahme erreicht werden, spricht man von der Thermoneutralzone (TNZ). In diesem Temperaturbereich fühlt sich ein Mensch behaglich. Es werden keine bewussten Temperaturempfindungen ausgelöst.

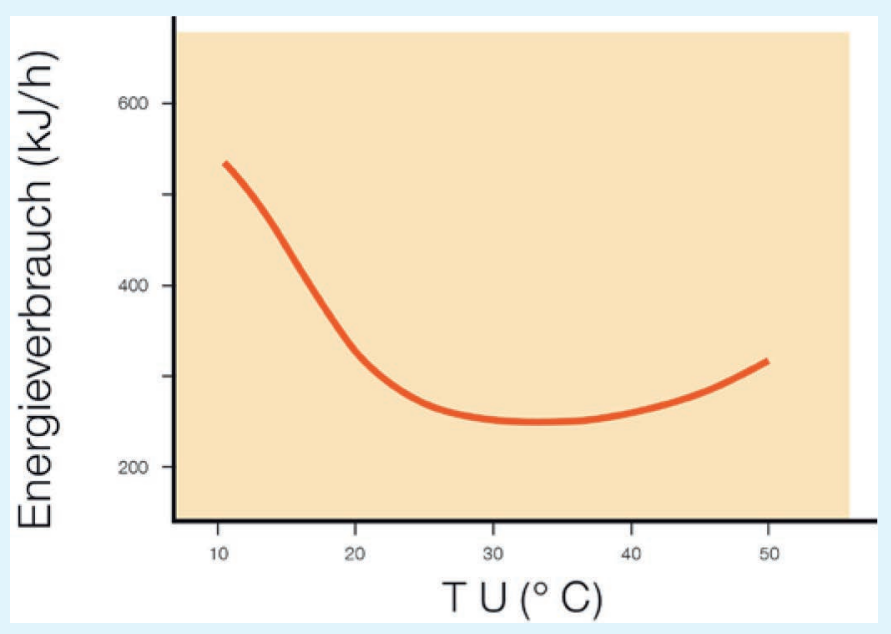

Abb. 6. Temperatur, Grundumsatz und Energieverbrauch (modifiziert nach $[1,13]$ ).

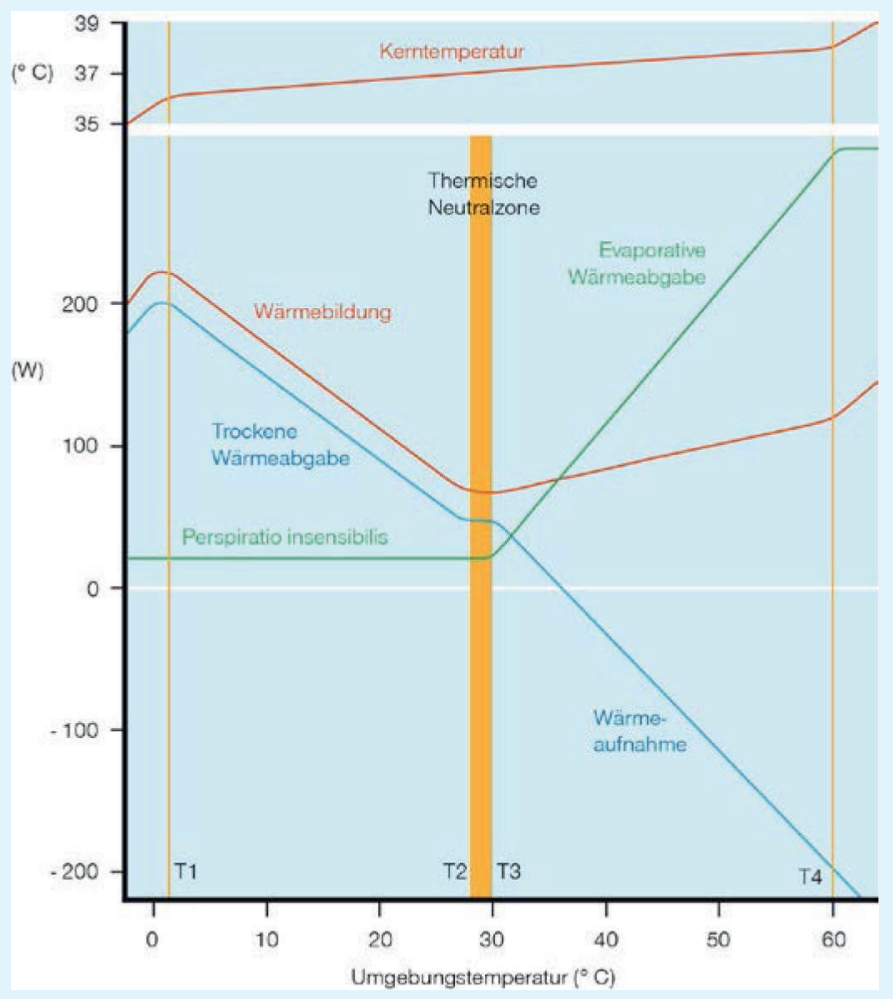

Abb. 7. Kerntemperatur, Wärmeaufnahme/-abgabe und Umgebungstemperatur (modifiziert nach $[1,13]$ ).
Für einen ruhenden, unbekleideten Menschen wird die TNZ mit einer Lufttemperatur von $27-31^{\circ} \mathrm{C}$ angegeben bei einer relativen Luftfeuchtigkeit von $50 \%$ und Windstille.

Jene Temperatur innerhalb der TNZ, bei der ein Minimum an Energie für die Wärmeregulation aufgewendet werden muss, bezeichnet man als Indifferenztemperatur. Bei diesen Bedingungen können Messungen des Grundumsatzes erfolgen (Abb. 6, 7).

Der genaue Wert der Indifferenztemperatur bzw. der Bereich der TNZ hängt von der Stoffwechsellage, dem Körperbau, der Körperoberfläche und dem Unterhautfettgewebe ab. Diese Werte differieren sowohl inter- als auch intraindividuell. In der Literatur wird die TNZ auch mit $27-35^{\circ} \mathrm{C}$ angegeben.

Der Bereich der TNZ wird ferner durch Luftbewegungen, Luftfeuchtigkeit, Luftdruck und Umgebungsstrahlung beeinflusst. Mit normaler Bekleidung sinkt der Bereich der TNZ auf $18-22{ }^{\circ} \mathrm{C}$. Im Wasser liegt die Indifferenztemperatur für einen ruhenden, unbekleideten Menschen bei $35-36^{\circ} \mathrm{C}$.

\section{Thermoregulation oberhalb oder} unterhalb der Thermoneutralzone

Liegen die Umgebungsbedingungen oberhalb bzw. unterhalb der TNZ, werden abgestuft die verschiedenen Thermoregulationsmechanismen aktiviert:

- Bei Hitze wird die Hautdurchblutung massiv erhöht. Die erhitze Haut wird gekühlt und gleichzeitig wird der Blutrückstrom zum Körperinnern reduziert, um einen Anstieg der Kerntemperatur zu vermeiden.

- Bei Kälte wird die Durchblutung zur Haut hin gedrosselt. Der Wärmeverlust an der Haut (z.B. InfrarotC-Abstrahlung) wird begrenzt und der Rückstrom abgekühlten Blutes vermindert.

Sowohl bei kühlen als auch bei warmen Umgebungsbedingungen (unterhalb oder oberhalb der TNZ) ist der Blutrückstrom aus der äusseren Körperschale (Haut, Schleimhaut) zum Körperkern zunehmend eingeschränkt.

Im Hinblick auf Wärmeanwendungen lässt sich für beide Situationen folgern, dass der Körperkern durch die Wärmeapplikation zunächst nicht erreicht wird.

\section{Disclosure Statement}

Die Firma Physiotherm ist seit 2006 Mandant von OP. Das Mandat erstreckt sich auf die medizinisch-wissenschaftliche Beratung.

TZ ist Physiker und seit 2007 Leiter der Abteilung für Forschung und Entwicklung der Firma Physiotherm.

Das Institut von WS war und ist Kooperationspartner im Rahmen klinischer Studien und Forschungsprojekte. 


\section{Literatur}

1 Gekle M, Singer D: Thermoregulation und Wärmehaushalt; in Klinke R, Pape HC Kurtz A, Silbernagel S (Hrsg): Physiologie. Stuttgart, Thieme, 2009, pp 500-515.

2 Koralewski HE, Gunga HC, Kirsch KA Wärmehaushalt und Temperaturregulation. Berlin, Charité, 2010.

3 Ulmer HV: Arbeits- und Umweltphysiologie; in Schmidt RF, Thews G (Hrsg): Phy siologie des Menschen. Berlin, Springer 1987, pp 683-716.

4 Vaupel P: Wärmehaushalt; in Thews G, Vaupel P (Hrsg): Vegetative Physiologie. Heidelberg, Springer, 2006, pp 322-343.

5 Gunga HC, Sandsund M, Reinertsen RE, Sattler F, Koch J: A non-invasive device to continuously determine heat strain in humans. J Therm Biol 2008:297-307.

6 Gunga HC, Werner A, Stahn A, Steinach M, Schlabs T, Koralewski E, Kunz D, Belavy DL, Felsenberg D, Sattler F, Koch J: The double sensor - a non-invasive device to continuously monitor core temperature in humans on earth and in space. Respir Physiol Neurobiol 2009;169(suppl 1):S63-68.

7 Opatz O, Stahn A, Werner A, Gunga HC: Determining core body temperature via heat flux - a new promising approach. Resuscitation 2010;81:1588-1589; author reply 1589.

8 Durrer B, Brugger H, Syme D; International Commission for Mountain Emergency Medicine: The medical on-site treatment of hypothermia: ICAR-MEDCOM recommendation. High Alt Med Biol 2003;4:99-103.

9 Harries M: Near drowning. BMJ 2003;327: 1336-1338.

10 Lang F, Lang P: Energie- und Wärmehaushalt; in Lang F, Lang P (Hrsg): Basiswissen Physiologie. Heidelberg, Springer, 2007, pp 181-190.
11 Ulmer HV: Energiehaushalt; in Schmidt RF, Thews G (Hrsg): Physiologie des Menschen. Berlin, Springer, 1987, pp 650-659.

12 Grissmer S: Arbeits-, Sport- und Leistungsphysiologie; Physiologie. Stuttgart, Thieme, 2010, pp 534-554.

13 Grissmer S: Energie- und Wärmehaus halt; Physiologie. Stuttgart, Thieme, 2010 pp 512-531.

14 Koralewski HE: Energiehaushalt und Temperaturregulation. Berlin, Charité, 2006.

15 Brück K: Wärmehaushalt und Temperaturregulation; in Schmidt RF, Thews G (Hrsg): Physiologie des Menschen. Berlin, Springer, 1987, pp 660-682.

16 Richter WS, Schmidt W: Bemerkungen zur Wirkungsweise von Infrarotkabinen. Dtsch Z Onkologie 2002;34:146-156. 